\title{
APLICAÇÕES DE REALIDADE VIRTUAL EM COORDENAÇÃO DE PROJETO DE ENGENHARIA CIVIL
}

\author{
Leopoldo Sousa $^{(2)}$, Rafael Cabral ${ }^{(3)}$, Rui Gavina ${ }^{(3)}$, Diogo Ribeiro ${ }^{(1)}$, Vitor Carvalho(3) \\ (1) CONSTRUCT-LESE, ISEP/ISEPBIM, Porto \\ (2) ISEP, Porto, Porto \\ (3) VN2R - Engineering Innovation Consulting, Lda, Porto
}

\begin{abstract}
Resumo
Nos dias de hoje as aplicações de Realidade Virtual são alvo de um interesse crescente por parte dos intervenientes do setor da Arquitetura, Engenharia, Construção e Operações (AECO). Para além da vertente de vendas e marketing, esta tecnologia tem vindo a ser explorada com sucesso na coordenação de projeto, permitindo aos intervenientes a visualização dos empreendimentos em ambiente virtual imersivo, com benefícios no que toca à sua compreensão visual e espacial, e acompanhamento nas várias fases do ciclo de vida. Neste âmbito, a Realidade Virtual surge como uma técnica complementar ao uso da metodologia Building Information Modeling (BIM), sobretudo como forma de agilizar a interação entre os diversos intervenientes e melhorando a deteção, interpretação e comunicação de incompatibilidades, sobretudo as de cariz funcional, e que não são facilmente detetáveis utilizando as tecnologias correntes. Neste enquadramento, o presente trabalho apresenta uma proposta para os principais requisitos que um software de Realidade Virtual deve possuir para a sua utilização em coordenação de projeto. Serão igualmente apresentados os resultados de uma análise comparativa do desempenho de alguns dos principais softwares disponíveis no mercado, estabelecendo-se uma matriz de funcionalidades de apoio na escolha da solução mais indicada.
\end{abstract}

\section{Introdução}

A quarta revolução industrial, também conhecida por Indústria 4.0, é caracterizada pela incorporação no processo produtivo de um conjunto de tecnologias disruptivas, que funcionam em sinergia, visando, entre outros, o aumento da eficiência produtiva e a customização da produção às necessidades do cliente. Um dos exemplos mais notórios de tecnologias emergentes, é a Realidade Virtual, amplamente utilizada nos dias de hoje em diversas indústrias e com crescente aplicação no setor AECO.

Os primeiros registos desta tecnologia são datados ao ano 1968, quando foram desenvolvidos os primeiros óculos de realidade virtual ou Head-Mounted Display (HDM). Este dispositivo seria o primeiro a permitir a interação com o ambiente virtual através de movimentos da cabeça. 
Os resultados, apesar de demonstrarem erros de visualização, foram bastante promissores uma vez que permitiram simular o efeito de imersão virtual tridimensional [1].

Os custos e a complexidade do equipamento, tornava-o inviável para a utilização industrial, no entanto, com a diminuição dos custos de fabrico, a tecnologia começou a disseminar-se, iniciando-se pela indústria dos jogos e proliferando para os demais setores [2]. No setor AECO a sua implementação iniciou-se sobretudo nas áreas de vendas e marketing, com enfoque na visualização geométrica e renderização de modelos virtuais.

O aprimoramento da tecnologia da Realidade Virtual, tanto em hardware quanto em software capazes de visualizar a geometria e os metadados, conjuntamente com a crescente adoção de novas metodologias, nomeadamente a metodologia BIM, fez com que o setor AECO ampliasse a sua utilização para as atividades de coordenação de projeto, embora a sua aplicação não seja ainda generalizada.

O uso de Realidade Virtual em coordenação de projeto possibilita uma deteção mais eficiente de incompatibilidades, nomeadamente as de caráter funcional, além de permitir o respetivo registo dentro do ambiente virtual. A deteção de eventuais incompatibilidades com base na sua visualização em ambiente virtual em reuniões de projeto, permitirá ainda melhorar a comunicação e coordenação entre especialidades. Por último, importa referir que as perceções espacial e de presença no interior do modelo, permitem simular a experiência de futuros utilizadores do empreendimento, ajudando o desenvolvimento do projeto e a realização de eventuais modificações.

\section{Realidade Virtual}

Até a década de 1980 a Realidade Virtual era definida apenas pela presença do usuário no ambiente virtual. Uma nova abordagem nesse ambiente foi iniciada no NASA Ames Research Center, denominado de "Virtual Interface Environment Workstation" a qual permite a interação do usuário com o ambiente virtual através do HDM interligado a luvas com sensores [3].

Essa nova abordagem, define a Realidade Virtual como um ambiente tridimensional gerado em computador na qual o utilizador integra-se no ambiente gerado ou é imerso nesse ambiente, podendo explorar e interagir com os elementos nele inseridos [4]. No entanto, o conceito de Realidade Virtual está dependente tanto da perceção sensorial pessoal como das tecnologias disponíveis capazes de gerar esses ambientes virtuais.

Quanto mais desenvolvidos forem os constituintes de um sistema de Realidade Virtual, maior será a imersão experienciada pelo utilizador. Os principais constituintes dos sistemas de Realidade Virtual são:

- HDM - estimulam os sentidos dos utilizadores através de ilusões de ótica de modo a transmitir a melhor representação de um mundo real;

- Aparelhos de Realidade Virtual (nomeadamente os comandos) - são produtos de hardware que permitem uma maior interação e exploração do ambiente virtual;

- Software- é o responsável pela gestão dos aparelhos constituintes do sistema analisando as informações recebidas e gerando os feedbacks esperados com o menor atraso possível;

- Áudio- não sendo tão relevante quanto a vertente visual, é de extrema importância na estimulação dos sentidos; 
- Sensores- compreender a fisiologia humana e a correta utilização dos sensores permitirá representar os movimentos reais no ambiente virtual.

O grau de imersão está diretamente relacionado com as especificações técnicas do HMD [5] relativas à imagem, som, sensores e processador (quer seja interno ou externo). Para que ocorra a imersão, a utilização de modelos digitais tridimensionais ou a sua renderização não é suficiente (como imagens $360^{\circ}$ ). Numa realidade imersiva a renderização deve ocorrer em tempo real, e ser visualizada e experienciada imersivamente em três dimensões, além de permitir a interação com os elementos construtivos [6].

Em relação às vantagens inerentes à Realidade Virtual, comparativamente com as imagens computorizadas, importa destacar a perceção espacial e o sentido de presença, permitindo simular a experiência de futuros utilizadores. A visualização de dados tridimensionais é outra grande vantagem, podendo-se analisar os elementos do modelo no interior do ambiente virtual. No setor AECO, a Realidade Virtual proporciona uma representação espaço-visual de um modelo projetado digitalmente e tem o potencial para se tornar uma ferramenta eficaz na exploração do projeto com os dados e parâmetros associados.

Existem indicadores baseados no uso corrente, tecnologia disponível e prospeção de mercado que demonstram o impacto que a Realidade Virtual tem nos workflows de coordenação. Esses indicadores demonstram que a implementação da tecnologia está fase crescente de maturação e que deverá atingir um uso generalizado entre 2021 e 2026 [7].

\section{Coordenação de Projeto}

A coordenação de projeto é uma atividade cada vez mais relevante no setor AECO dada a crescente complexidade interdisciplinar, impulsionada pelos recentes avanços tecnológicos, desenvolvimento de legislação aplicável a novas especialidades (ex. segurança contra incêndio) ou reestruturação das existentes (ex. térmica e acústica), economia atual, entre outros. $\mathrm{O}$ desempenho da coordenação de projeto é determinante para a redução de erros e eficácia na execução em obra. Cada empreendimento requer a realização de diversos projetos, em diferentes períodos do ciclo de vida, e em que a visão de cada interveniente está frequentemente limitada pela tarefa que desempenha [8].

A finalidade da coordenação de projeto, segundo a Lei $n^{\circ} .40 / 2015$ de 01 de Junho [9] é coordenar e compatibilizar as diferentes especialidades, estabelecer a comunicação entre os intervenientes, fornecer em tempo útil as alterações necessárias e garantir a continuidade entre projetos ao longo do desenvolvimento do empreendimento de acordo com as disposições legais e regulamentares aplicáveis. O cumprimento integral destes desígnios tem vindo a ser um grande desafio dada a complexidade das atividades de coordenação de projeto.

É neste âmbito que se têm observado os benefícios da utilização da metodologia BIM apesar de alguma ineficiência pontual quanto aos aspetos comunicacionais e à deteção de erros, sobretudo os de cariz funcional [10]. O Coordenador de Projeto, através dos fluxos de coordenação BIM, será preferencialmente o responsável pela receção dos modelos das especialidades, centralizá-las no modelo federado e gerir a clash detection assegurando o cumprimento da legislação. É aconselhada a presença dos projetistas e outros intervenientes nas reuniões de coordenação com recurso à visualização virtual onde será apresentado o relatório afeto às incompatibilidades e definidas as possíveis alterações. Os modelos deverão ser 
atualizados pelos projetistas de acordo com o relatório elaborado pelo coordenador de projeto. Este é um processo iterativo a realizar periodicamente que só deverá terminar quando não existirem mais incompatibilidades, tal como demonstrado na Figura 1 [11].

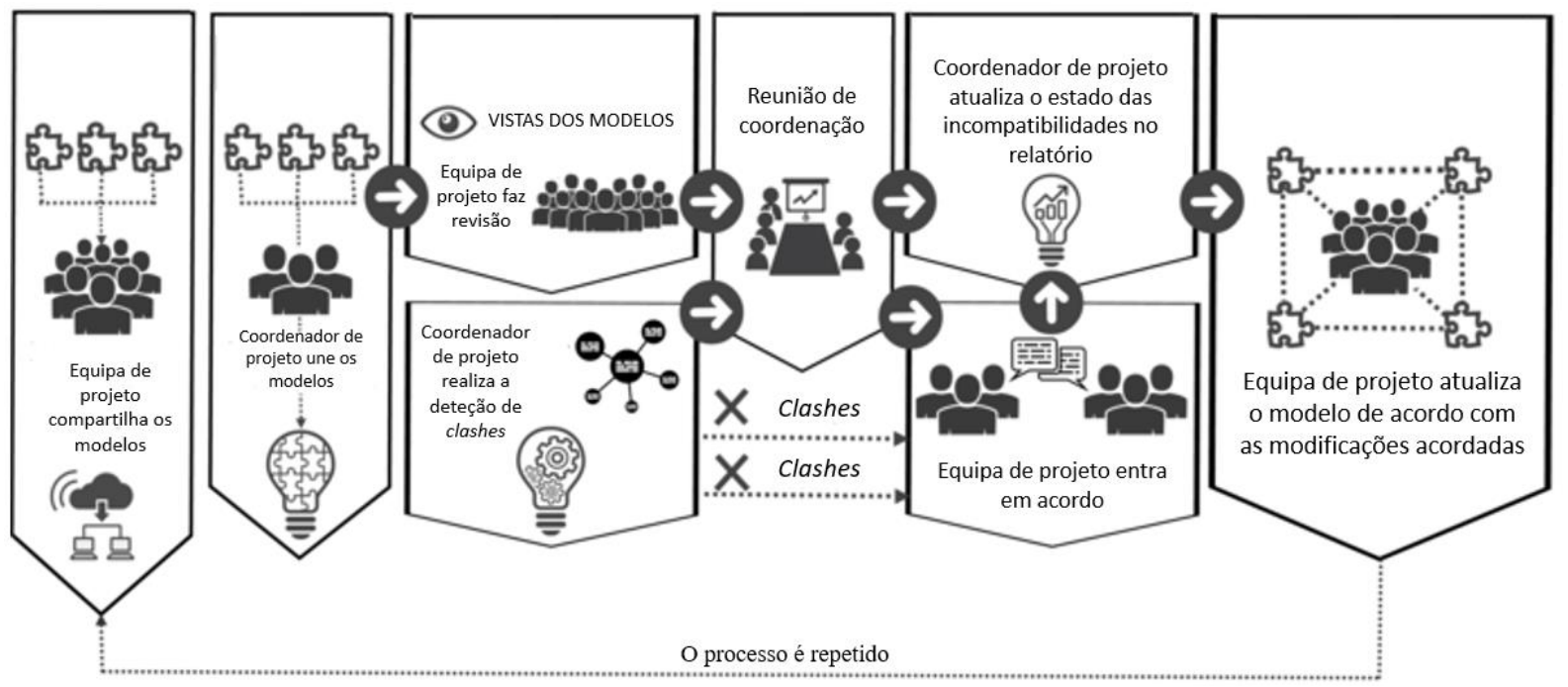

Figura 1: Workflow de Coordenação de Projeto (adaptado de [11]).

Durante o ciclo de vida de um empreendimento é frequente ocorrerem reuniões de coordenação de projeto, desde reuniões de revisão, de planeamento, etc. Essas reuniões podem ser realizadas em diferentes formatos, tais como, reuniões presenciais, reuniões de grupo em ambiente virtual (mesmo espaço físico), ou ainda colaboração remota em ambientes virtuais distribuídos espacialmente.

Os desafios destas reuniões poderão advir de [11]:

- Experiência e competência dos intervenientes: por mais poderosas que sejam as ferramentas e os softwares ao dispor de uma equipa, é necessário que esta possua qualificação e valias suficientes para a obtenção dos melhores resultados;

- Barreira comunicacional: a especialização de cada interveniente na sua disciplina dificulta a transmissão de conhecimento e informação aos restantes membros de diferentes áreas.

No caso de haver necessidade da realização de reuniões remotas, as dificuldades comunicacionais são acrescidas. Uma reunião de colaboração em ambiente virtual deve oferecer acesso simultâneo multiutilizador a um sistema de Realidade Virtual que permita realizar trabalho cooperativo, ou poderá ainda ser liderado pelo Coordenador de Projeto, acompanhado pelos restantes intervenientes visualizando via desktop ou HDM de visualização (sem comandos).

A recente situação pandémica tornou evidente as debilidades e fragilidades das reuniões de coordenação não presenciais, tendo sido verificado um aumento expressivo pela procura de serviços de equipamentos de Realidade Virtual no setor AECO [12]. 


\section{Realidade Virtual em Coordenação de Projeto}

A Realidade Virtual em coordenação de projeto é integrada na metodologia BIM, sendo recomendado que todos os intervenientes a utilizem como meio de coordenação virtual e de desenvolvimento do projeto [13]. Deste modo, será possível agregar os modelos digitais das diversas especialidades num modelo federado, permitindo a compatibilização e a eficiente coordenação de projeto. A estratégia de federação, embora não seja estritamente necessária, é considerada como boa prática para manter as responsabilidade e autoria inerentes a cada projeto aos seus autores [14].

Através da metodologia BIM, muitas das incompatibilidades, erros de projeto e omissões são detetados automaticamente. A Realidade Virtual será uma ferramenta bastante útil para o coordenador de projeto em que, através da presença e interação num ambiente virtual imersivo, conseguirá detetar com muito maior facilidade as incompatibilidades funcionais. Tal é possível pela sensação geral de desconforto, derivada da experiência em primeira pessoa na utilização do edifício, como as demonstradas na Figura 2.

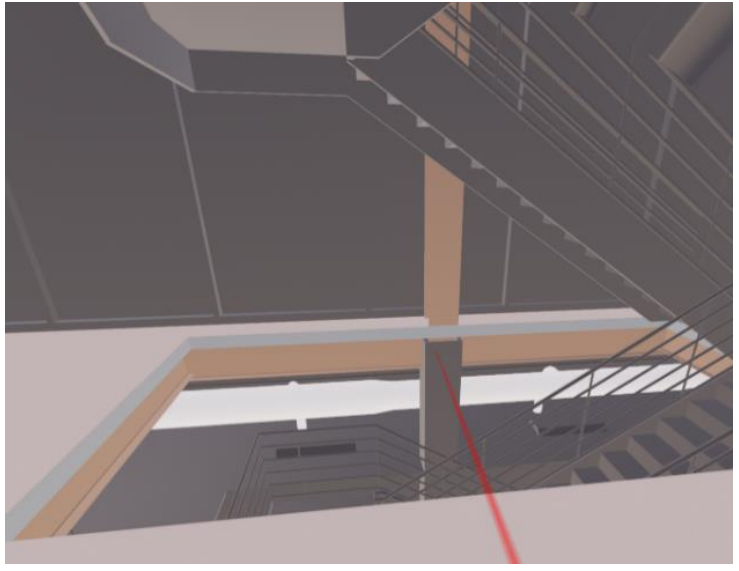

a)

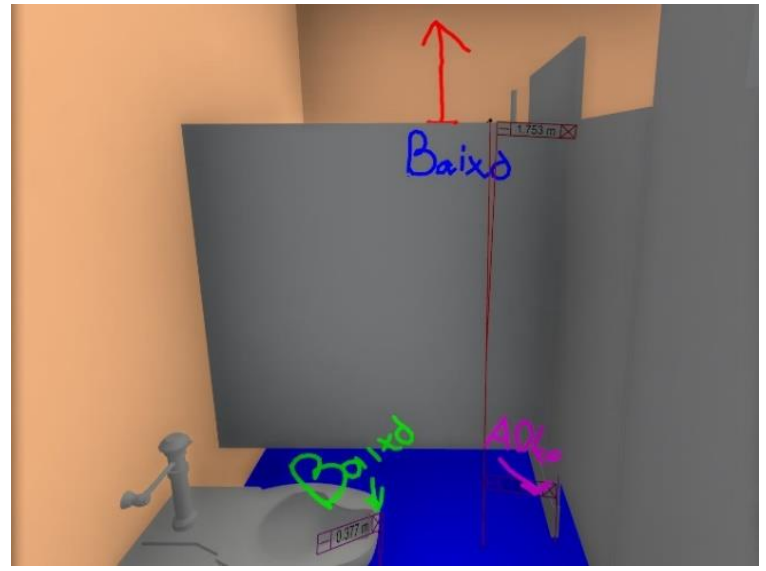

b)

Figura 2: Exemplos de erros detetados em Ambiente Virtual imersivo: a) ausência de guardas de segurança numa caixa de escadas (utilizando o EnscapeVR); b) dimensões inadequadas para uma instalação sanitária (utilizando BIMXplorer).

A Realidade Virtual é também uma mais valia para os projetistas e para o cliente nas reuniões de projeto, nomeadamente na comunicação de problemas e soluções. No decorrer das reuniões de coordenação, a visualização destes problemas com maior realismo irá auxiliar as tomadas de decisão relativamente às possíveis soluções. No entanto, em muitas situações, as reuniões podem focar-se em aspetos não essenciais que não fazem parte dos objetivos inicialmente propostos [10]. Tal é possível pelo aumento da eficiência comunicacional entre os diferentes intervenientes, essencial para a redução de desvios entre a fase projeto e a fase de execução [15]. O acompanhamento da reunião poderá ser feito através da presença no ambiente virtual em simultâneo (utilizando um HDM ou versão desktop), conforme exemplificado na Figura 3, ou pela transmissão da vista do coordenador de projeto nesse ambiente. Caso se pretenda a visualização utilizando um HDM, a sua utilização não deverá ser muito prolongada de forma a diminuir a probabilidade de cybersickness. Este fenómeno é caracterizado como um sentimento de desconforto derivado da exposição à Realidade Virtual e que está associado a sintomas como náuseas, desorientação e distúrbios oculomotores. 


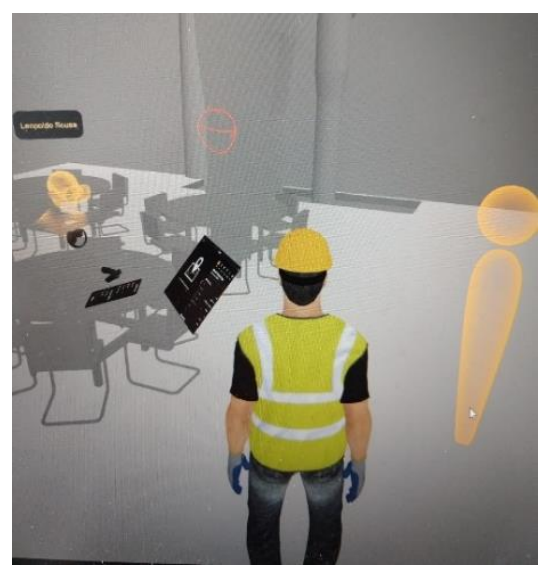

a)

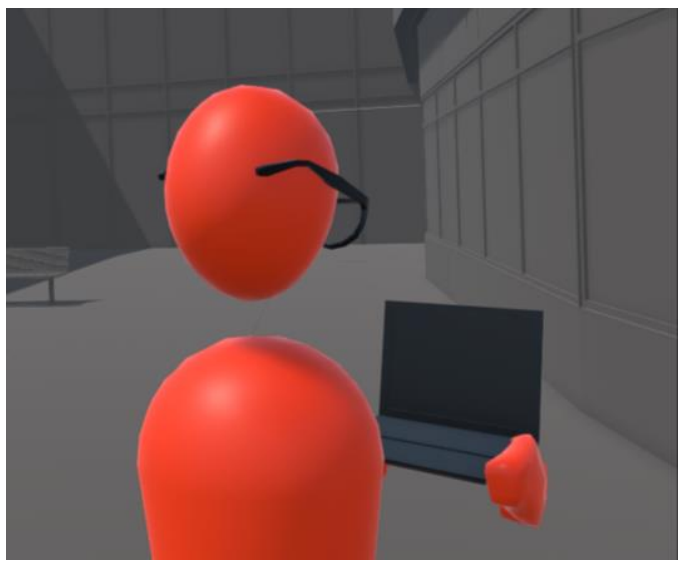

b)

Figura 3: Simulação de Reunião Multiutilizador: a) com participantes em Ambiente Virtual imersivo e desktop (acervo dos autores utilizando VRcollab); b) com participantes em desktop (acervo dos autores utilizando IrisVR).

De acordo com [16], os registos e documentação são um dos principais fatores que influenciam o desempenho em coordenação. Tanto na fase de deteção de erros como durante as reuniões de coordenação devem ser tomadas as devidas anotações para posterior documentação e revisão. A possibilidade de criar anotações dentro do ambiente virtual imersivo é uma característica bastante importante nos softwares de Realidade Virtual. A falta ou inadequação de informação será bastante prejudicial para a comunicação. Os meios mais comuns de documentação nestes ambientes são: fotografias, comentários ou representações gráficas (markups) conforme ilustrado na Figura 4.

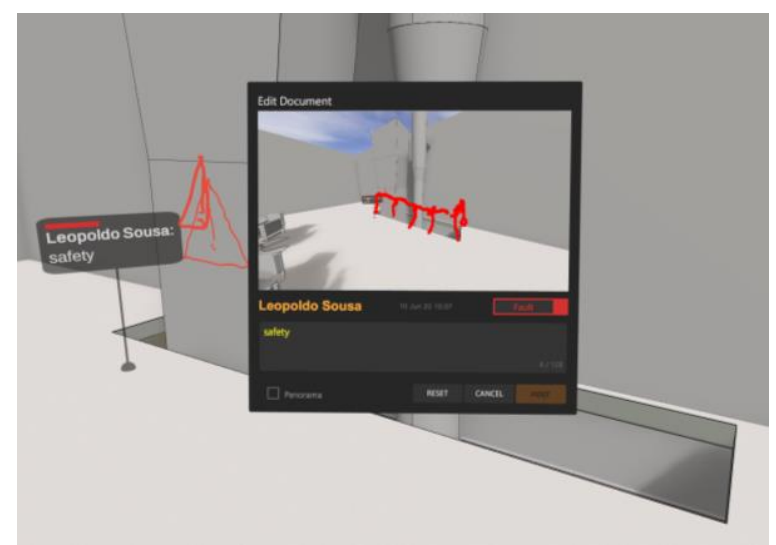

Figura 4: Anotações através de fotografia, comentário e markup (acervo dos autores utilizando o VRcollab).

Acrescenta-se ainda o potencial da Realidade Virtual para desenvolvimento do projeto pela experiência do utilizador. A experiência pessoal durante a navegação em ambientes imersivos gera um aumento de empatia relativamente aos outros modos de visualização. Existe, por exemplo, a possibilidade de diminuir a altura de visualização simulando o ponto de vista de uma criança, ou mesmo experienciar o percurso de acesso a uma instalação sanitária por um utilizador em cadeira de rodas [17], conforme demonstrado na Figura 5. 


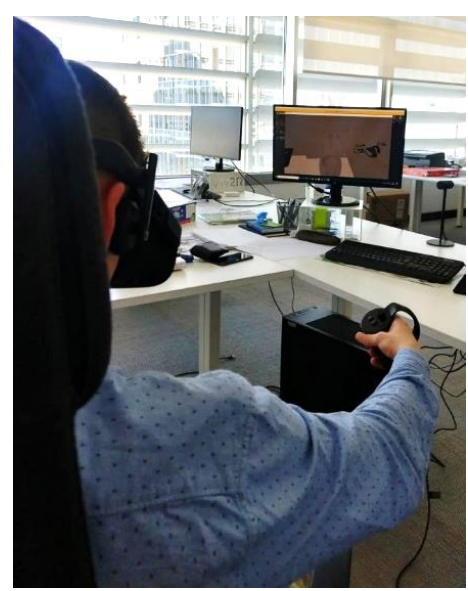

Figura 5: Simulação de utilização de uma instalação sanitária por utilizador em cadeira de rodas (acervo dos autores).

\section{Requisitos e Soluções}

Nesta secção serão detalhados os requisitos para os softwares de Realidade Virtual, além de uma análise comparativa de diferentes soluções no que toca à sua adequabilidade à Realidade Virtual em coordenação de projeto.

A utilização de Realidade Virtual para efeitos de coordenação de projeto está dependente dos softwares existentes e das suas funcionalidades. Existem no mercado soluções que permitem a sincronização com plataformas de coordenação BIM, plug-ins nos softwares nativos ou importação dos formatos usuais como .IFC, .OBJ e .FBX. Desta forma é possível ultrapassar os processos morosos e dispendiosos de programação dos ambientes virtuais nos tradicionais game engines, que apesar de serem bastante customizáveis, não são ideais caso seja necessário efetuar uma grande quantidade de alterações ou em que estas sejam de elevado grau de complexidade [13].

Para a seleção do software adequado num projeto específico foram definidos os seguintes requisitos de utilização:

- Ver metadata- é necessário ter acesso às informações não geométricas dos elementos. É exemplo disso a indicação das dimensões, inclinação e sistema de uma tubagem que se encontra a obstruir a visão de um posto de controlo numa fábrica;

- Ferramentas de medição- esta ferramenta permite perceber se são cumpridos os requisitos funcionais impostos pelo cliente tais como o espaço de circulação de um corredor, o pé direito na circulação de uma escada, etc.;

- Desligar/Ligar layers e elementos- é necessário quando se pretende visualizar elementos que se encontram ocultos por outra especialidade para, por exemplo, verificar a disposição dos elementos de AVAC ou hidráulicos no interior de um teto falso. Poderá ser colmatado com visão X-Ray quando apenas se pretenda visualização, mas não interação com os elementos;

- Anotações e Screenshots- é necessário marcar ou catalogar os pontos de interesse no registo das incompatibilidades de uma forma consistente e organizada, sem que seja necessário sair do ambiente virtual, para auxiliar a revisão nas reuniões de projeto; 
- Multiutilizador- nos casos de coordenação colaborativa é importante que a equipa de coordenação possa fazer uma análise em simultâneo, mesmo que remotamente, colocando ao corrente toda a equipa de projeto;

- Exportação de Anotações ou Screenshots- considerando a parte de documentação, especialmente em modelos de dimensão considerável, deverá ser possível extrair as anotações e screenshots de modo a manter o registo das ações realizadas em ambiente virtual;

- Planos de Corte- auxiliam a visualização do modelo em escala reduzida, escala real e teletransporte através de secções, sendo disto exemplo a visualização de todos os fogos num determinado piso de habitação;

- Walk Mode- permite simular o movimento real no interior do modelo para auxiliar a perceção espacial e experienciar o percurso de um possível utilizador, sendo disto exemplo a verificação dos percursos entre postos de trabalho de uma unidade de produção.

Para o estudo e experimentação, o HDM utilizado foi o Oculus Rift ligado a um computador com processador Intel Core i5-9600k CPU com 16.0GB de RAM e uma placa gráfica NVIDIA GeForce GTX 1050 Ti.

Foi utilizado um modelo fictício federado modelado no software Revit de um hospital constituído por 10 modelos de diferentes especialidades, unificados através dos respetivos links ilustrados na Figura 6. De acordo com [18], devemos importar para o ambiente virtual um número total de polígonos de 2000000 e de polígonos por malha de 65000 , no entanto, tal não será abordado no presente trabalho.

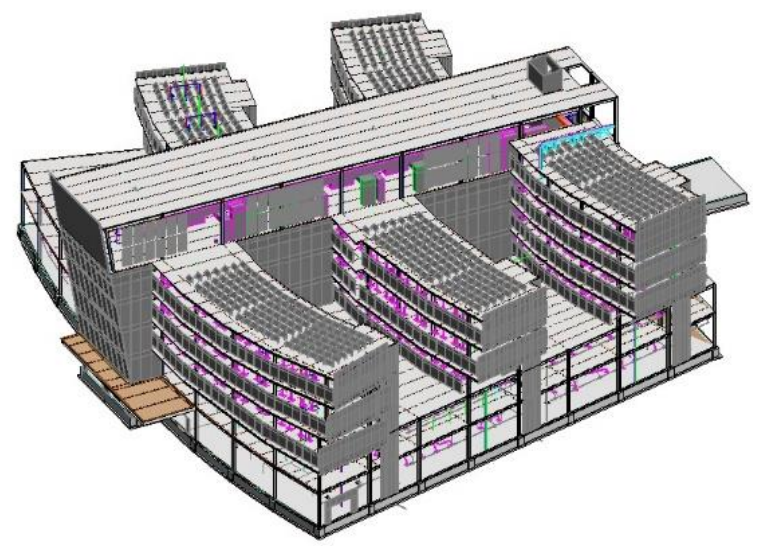

a)

\begin{tabular}{l}
$\qquad$ Link Name \\
\hline Autodesk_Hospital_Metric_Architectural_Central.rvt \\
\hline Autodesk_Hospital_Metric_Electrical_Central.rvt \\
\hline Autodesk_Hospital_Metric_FireAlarm_Central.rvt \\
\hline Autodesk_Hospital_Metric_Mechanical_Central.rvt \\
\hline Autodesk_Hospital_Metric_Plumbing_Central.rvt \\
\hline Autodesk_Hospital_Metric_Site Logistics.rvt \\
\hline Autodesk_Hospital_Metric_Site.rvt \\
\hline Autodesk_Hospital_Metric_Sprinkler_Central.rvt \\
\hline Autodesk_Hospital_Parking Garage.0001.rvt \\
\hline Autodesk_Hospital_Parking Garage.rvt \\
\hline
\end{tabular}

b)

Figura 6: Modelos utilizados para testes: a) modelo federado; b) listagem dos modelos das especialidades.

Neste âmbito procedeu-se à experimentação empírica de 9 softwares, sendo eles ou não direcionados especificamente para integração de Realidade Virtual em coordenação de projeto. A importação do modelo foi direta, ou seja, não se recorreu qualquer tipo de programação ou customização no software e no modelo.

Numa primeira fase foram analisadas as interfaces do utilizador, verificando os diferentes comandos, funcionalidades e modos de utilização. Posteriormente, na fase de validação os requisitos foram aplicados no contexto de coordenação de projeto, procurando identificar e 
documentar incompatibilidades. Na Tabela 1 ilustra-se por meio de uma matriz a correspondência entre os vários softwares e as suas funcionalidades no âmbito da coordenação de projeto.

Tabela 1: Funcionalidades dos softwares de Realidade Virtual no âmbito da coordenação de projeto (verificados a junho de 2020)

\begin{tabular}{|c|c|c|c|c|c|c|c|c|c|}
\hline & $\begin{array}{c}\text { Arkio } \\
\text { (v. Beta) }\end{array}$ & BIMXplorer & $\begin{array}{c}\text { Dimension } \\
10 \\
\end{array}$ & Enscape & \begin{tabular}{|c|} 
Insite \\
VR
\end{tabular} & $\begin{array}{l}\text { Iris } \\
\text { VR }\end{array}$ & Revizto & $\begin{array}{c}\text { VR } \\
\text { Collab }\end{array}$ & VREX \\
\hline Ver Metadata & & $\mathrm{x}$ & $x$ & & $*$ & $x$ & $x$ & $\mathrm{x}$ & $x$ \\
\hline $\begin{array}{l}\text { Ferramentas de } \\
\text { Medição }\end{array}$ & $x$ & $\mathrm{x}$ & $x$ & & $\mathrm{x}$ & $x$ & & $\mathrm{x}$ & $x$ \\
\hline $\begin{array}{l}\text { Desligar/Ligar } \\
\text { Layers }\end{array}$ & & $* *$ & & & $x$ & $x$ & & $x$ & $* *$ \\
\hline $\begin{array}{l}\text { Desligar/Ligar } \\
\text { Elementos }\end{array}$ & & $\mathrm{x}$ & $\mathrm{x}$ & & & $x$ & & $x$ & $x$ \\
\hline Anotações & $x$ & & $x$ & & $\mathrm{x}$ & **** & & $x$ & $x$ \\
\hline Screenshots & & $x$ & $x$ & $x$ & $x$ & $x$ & & $x$ & $x$ \\
\hline Multiutilizador & $x$ & & $x$ & & $x$ & $x$ & & $x$ & $x$ \\
\hline $\begin{array}{l}\text { Exportação de } \\
\text { anotações/scre- } \\
\text { enshots }\end{array}$ & $* * *$ & & $\mathrm{x}$ & & $x$ & $x$ & & $\mathrm{x}$ & $x$ \\
\hline $\begin{array}{l}\text { Localização das } \\
\text { anotações }\end{array}$ & & & $\mathrm{x}$ & & $x$ & $\mathrm{x}$ & & $\mathrm{x}$ & $x$ \\
\hline $\begin{array}{l}\text { Um plano de } \\
\text { corte }\end{array}$ & $x$ & $\mathrm{x}$ & $x$ & & $x$ & $x$ & & $x$ & $x$ \\
\hline $\begin{array}{l}\text { Section Box ou } \\
\text { mais de dois pla- } \\
\text { nos de corte }\end{array}$ & & & & & & & & $\mathrm{x}$ & $x$ \\
\hline Walk Mode & & $\mathrm{x}$ & $\mathrm{x}$ & $x$ & $\mathrm{x}$ & $x$ & $x$ & $x$ & $x$ \\
\hline
\end{tabular}

A versão beta do software Arkio é especializada na criação de modelos conceptuais no interior do ambiente virtual. Apesar de apresentar alguns dos requisitos definidos, é um software direcionado á vertente da arquitetura.

Tanto o Enscape como o Revizto apenas permitiram a visualização e movimento no interior do ambiente virtual. O Revizto permitia a visualização de incompatibilidades previamente identificadas bem como os metadados, no entanto, de forma pouco prática, visto que as janelas do software no computador eram visualizadas no ambiente virtual sem que fosse possível interagir diretamente.

O BIMXplorer é um software de utilização bastante simples e intuitiva com uma grande margem de desenvolvimento, parecendo mais direcionado para a deteção de incompatibilidades no ambiente virtual do que para reuniões de coordenação.

Os softwares Dimension 10, Insite VR, Iris VR, VR Collab e VREX são os mais destinados a coordenação de projeto, incluindo a integração em plataformas BIM e a identificação de incompatibilidades.

\section{Conclusões e desenvolvimentos futuros}

A tecnologia da Realidade Virtual aplicada à coordenação de projetos permite otimizar os processos colaborativos, detetar incongruências e omissões em fase de projeto e melhorar a 
comunicação e interpretação entre os diversos intervenientes. Esta tecnologia requer treino e experiência, permitindo que qualquer interveniente, independentemente do seu background, consiga interpretar com facilidade o problema ou solução apresentada, sem a necessidade de desenhos 2D ou modelos 3D.

Os hardwares e softwares atualmente disponíveis no mercado, fornecem às empresas ferramentas e soluções economicamente acessíveis, o que justifica a crescente implementação da tecnologia no sector AECO. Apresentando-se como uma tecnologia complementar aos processos já existentes, com os respetivos custos e trabalho associados, a sua utilização requer estudos de viabilidade para o retorno do investimento. O software poderá ser analisado por projeto, utilizações pontuais para vários utilizadores (através de licenças coletivas). Já o hardware consiste num investimento de médio ou longo prazo, podendo não justificar a aquisição por parte de outros intervenientes que não sejam responsáveis pela coordenação de projeto (como o cliente ou os projetistas). Devem também ser evitadas quaisquer partilhas de material derivado à situação pandémica atual pelo que o hardware deverá ser pessoal e intransmissível.

A avaliação da relação custo-benefício para o uso da Realidade Virtual em coordenação de projeto, deve, resumidamente, seguir os seguintes pressupostos de escolha de hardware, software e modelo de licenciamento:

1. Definir as funcionalidades pretendidas consoante o caso de estudo em questão a partir de uma previsão dos erros expectáveis a serem identificados;

2. Planear o número de utilizações individuais (coordenador de projeto) para deteção de erros;

3. Planear o número e duração de reuniões mensais ou anuais;

4. Definir um custo/hora base em função das utilizações do coordenador de projeto, outros intervenientes (caso se pretenda multiutilizador) e utilização da Realidade Virtual para outros fins (como vendas e marketing);

5. Verificar quais os softwares que possuem os requisitos, verificando o custo/hora dos mesmos em função da periodicidade da licença (mensal, anual, etc.) e das horas de utilização;

6. Atendendo a que o coordenador de projeto deve possuir um HMD que permita interação, deve ser estabelecido o modo de visualização e interação dos restantes intervenientes em reunião, averiguando o hardware disponível, ou a possível aquisição do mesmo caso se justifique para utilizações futuras.

No decorrer desta pesquisa foi constatado que a Realidade Virtual poderá ser utilizada para visualização e auxílio das tomadas de decisão em relação a conflitos previamente identificados em softwares de coordenação. O estudo a ser desenvolvido futuramente passará pela alocação de informação a essas clashes (em formato .BCF, por exemplo), posterior alocação no ambiente virtual, e definição das vias possíveis de importação.

\section{Agradecimentos}

Os autores expressam o seu agradecimento a todas as empresas e instituições pelo fornecimento gratuito da demonstração, licença de estudante ou versão beta dos softwares. 


\section{Referências}

[1] Sutherland, I. (1968). A head-mounted three dimensional display. Fall Joint Computer Conference. University of Utah, Salt Lake City, Utah.

[2] Liagkou, V., Salmas, D., Stylios, C. (2018). Realizing Virtual Reality Learning Environment for Industry 4.0. $12^{\text {th }}$ CIRP Conference on Intelligent Computation in Manufacturing Engineering. Nápoles.

[3] NASA (2014). The Virtual Interface Environment Workstation (VIEW) [Available Online: https://www.nasa.gov/ames/spinoff/new_continent_of_ideas/].

[4] Virtual Reality Society (VRS). What is Virtual Reality? [Available Online: https://vrs.org.uk/virtual-reality/what-is-virtual-reality.html].

[5] Dörner, R., Brot, W., Grimm, P., Jung, B. (2013). Virtual and Augmented Reality (VR/AR): Grundlagen und Methoden der Virtuellen und Augmentierten Realität. Springer. eBook ISBN: 978-3-642-28903-3.

[6] Du, J., Zou, Z., Shi, Y., Zhao, D. (2018). Zero latency: Real-time synchronization of BIM data in virtual reality for collaborative decision-making. Automation in Construction, 85: 51-64.

[7] Gartner Inc. (2016). Hype Cycle for Emerging Technologies Identifies Three Key Trends That Organizations Must Track to Gain Competitive Advantage, Press Release (disponível online).

[8] Kubicki, S., Bignon, J.-C., Halin, G., Humbert, P. (2006). Assistance to Building Construction Coordination - Towards a Multi-View. Journal of Information Technology in Construction, 11: 565-586.

[9] Decreto Lei no 40/2015 de 1 de Junho da Assembleia da República: I série, 3373 - 3441. Acedido a 06 ago. 2020. Disponível em www.dre.pt.

[10] Anderson, A., Dossick, C., Azari, R., Taylor, J., Hartmann, T., Mahalingham, A. (2014). Exploring BIMs as Avatars: Using 3D Virtual Worlds to Improve Collaboration with Models. ASCE Construction Research Congress.

[11]Pärn, E., Edwards, D., Sing, M. (2018). Origins and Probabilities of MEP and Structural Design Clashes within a Federated BIM. Automation in Construction, 85: 209-219.

[12] Rubenstone, J. (2020). Turning to Virtual Reality BIM Review During a Pandemic. Engineering News-Record.

[13]Zaker, R., Coloma, E. (2018). Virtual reality-integrated workflow in BIM enabled projects collaboration and design review: a case study. Visualization in Engineering, 6:4.

[14] Preidel, C., Borrmann, A., Oberender, C., Tretheway, M. (2016). Seamless integration of common data environment access into BIM authoring applications: The BIM integration framework. In eWork and eBusiness in Architecture, Engineering and Construction: ECPPM. Limassol.

[15] Gamil, Y., Rahman, I. (2017). Identification of Causes and Effects of Poor Communication in Construction Industry: A Theoretical Review. Emerging Science Journal, 1(4): 239-247.

[16] Alaloul, W., Liew, M., Zawawi, N. (2016). Identification of coordination factors affecting building projects performance. Alessandria Engineering Journal, 55(3): 2689-2698.

[17] Purcell, J., Bulfinch, S. (2019). VR for Knowledge Transfer and Teaching Empathic Design. $\quad$ Digital Built Week NA 2019. Seattle. 
[18] Pybus, C., Graham, K., Doherty, J., Arellano, N., Fai, S. (2019). New realities for Canada's parliament: a workflow for preparing heritage bim for game engines and virtual reality, Int. Arch. Photogramm. Remote Sens. Spatial Inf. Sci., XLII-2/W15, 945-952. 